\title{
Mutation in TORIAIP1 encoding LAP1B in a form of muscular dystrophy: A novel gene related to nuclear envelopathies
}

\author{
Gulsum Kayman-Kurekci ${ }^{\mathrm{a}}$, Beril Talim ${ }^{\mathrm{b}}$, Petek Korkusuz ${ }^{\mathrm{c}}$, Nilufer Sayar ${ }^{\mathrm{d}}$, \\ Turkan Sarioglu $^{\mathrm{e}}$, Ibrahim Oncel ${ }^{\mathrm{f}}$, Parisa Sharafi ${ }^{\mathrm{a}}$, Hulya Gundesli ${ }^{\mathrm{a}, 1}$, \\ Burcu Balci-Hayta ${ }^{\mathrm{a}}$, Nuhan Purali ${ }^{\mathrm{g}}$, Piraye Serdaroglu-Oflazer ${ }^{\mathrm{h}}$, \\ Haluk Topaloglu ${ }^{\mathrm{f}}$, Pervin Dincer ${ }^{\mathrm{a}, *}$ \\ ${ }^{a}$ Department of Medical Biology, Hacettepe University, Faculty of Medicine, Sihhiye, 06100 Ankara, Turkey \\ ${ }^{\mathrm{b}}$ Department of Pediatrics, Pathology Unit, Hacettepe University, Faculty of Medicine, Sihhiye, 06100 Ankara, Turkey \\ ${ }^{\mathrm{c}}$ Department of Histology and Embryology, Hacettepe University, Faculty of Medicine, Sihhiye, 06100 Ankara, Turkey \\ ${ }^{\mathrm{d}}$ Department of Molecular Biology and Genetics, Bilkent University, Faculty of Science, Bilkent, 06800 Ankara, Turkey \\ ${ }^{\mathrm{e}}$ Department of Histology and Embryology, Istanbul University, Istanbul Medical Faculty, Capa, 34093 Istanbul, Turkey \\ ${ }^{\mathrm{f}}$ Department of Pediatrics, Neurology Unit, Hacettepe University, Faculty of Medicine, Sihhiye, 06100 Ankara, Turkey \\ ${ }^{\mathrm{g}}$ Department of Biophysics, Hacettepe University, Faculty of Medicine, Sihhiye, 06100 Ankara, Turkey \\ ${ }^{\mathrm{h}}$ Department of Neurology, Istanbul University, Istanbul Medical Faculty, Capa, 34093 Istanbul, Turkey
}

Received 4 March 2014; received in revised form 21 April 2014; accepted 24 April 2014

\begin{abstract}
We performed genome-wide homozygosity mapping and mapped a novel myopathic phenotype to chromosomal region 1q25 in a consanguineous family with three affected individuals manifesting proximal and distal weakness and atrophy, rigid spine and contractures of the proximal and distal interphalangeal hand joints. Additionally, cardiomyopathy and respiratory involvement were noted. DNA sequencing of torsinA-interacting protein 1 (TORIAIPI) gene encoding lamina-associated polypeptide 1B (LAP1B), showed a homozygous c.186delG mutation that causes a frameshift resulting in a premature stop codon (p.E62fsTer25). We observed that expression of LAP1B was absent in the patient skeletal muscle fibres. Ultrastructural examination showed intact sarcomeric organization but alterations of the nuclear envelope including nuclear fragmentation, chromatin bleb formation and naked chromatin. LAP1B is a type-2 integral membrane protein localized in the inner nuclear membrane that binds to both A- and B-type lamins, and is involved in the regulation of torsinA ATPase. Interestingly, luminal domain-like LAP1 (LULL1) - an endoplasmic reticulum-localized partner of torsin A-was overexpressed in the patient's muscle in the absence of LAP1B. Therefore, the findings suggest that LAP1 and LULL1 might have a compensatory effect on each other. This study expands the spectrum of genes associated with nuclear envelopathies and highlights the critical function for LAP1B in striated muscle.
\end{abstract}

(c) 2014 Elsevier B.V. All rights reserved.

\footnotetext{
* Corresponding author. Tel.: +90 312305 2541; fax: +90 3123096060 E-mail address: pdincer@hacettepe.edu.tr (P. Dincer).

${ }^{1}$ Present address: Program in Gene Function and Expression, University of Massachusetts Medical School, Worcester, MA, USA.
}

\section{Introduction}

Muscular dystrophies are a clinically and genetically heterogeneous group of hereditary muscle disorders characterized by progressive muscular degeneration and 
weakness [1]. An important group of muscular dystrophies is limb-girdle muscular dystrophies, which predominantly present with proximal weakness with sparing of the facial and distal muscles, and cardiomyopathy in some subtypes [2]; however even when the disease begins with limb-girdle weakness, additional clinical features lead to consider other myopathic disorders. When limb-girdle weakness is combined with early distal muscle involvement, contractures and cardiac involvement, the phenotype is usually categorized as Emery-Dreifuss muscular dystrophy (EDMD) [3,4]. On the other hand, additional characteristic features of the phenotype such as early and non-proportional weakness, and atrophy of the distal muscles, are indicative of myopathies with predominantly distal muscle involvement $[5,6]$. To date, some patients are affected with muscular dystrophy of unknown genetic aetiology, and some families are not linked to any of the known loci.

Lamina-associated polypeptide 1s (LAP1s) were originally identified as three integral membrane proteins (LAP1A, LAP1B and LAP1C) specific to the nuclear envelope in the rat liver [7]. To date, TORIAIPI (torsinA-interacting protein 1 gene) encoding LAP1B protein is the only cloned human lamina-associated polypeptide 1 [8]. Despite the fact that the function of LAP1B is currently not fully known, this type-2 integral membrane protein located in the inner nuclear membrane is known to bind both A- and B-type lamins [7-10]. Subsequently, LAP1 has been implicated in the regulation of torsinA ATPase which is known to cause the severe movement disorder DYT1 dystonia (MIM 128100) $[11,12]$. Another torsinA-interacting protein, luminal domain-like LAP1 (LULL1) encoded by TOR1AIP2 is located in the endoplasmic reticulum and shares homologous C-terminal regions with LAP1 allowing interaction with torsinA. These two proteins appear to have arisen from a gene duplication [11]. LAP1B is widely expressed in a variety of tissues, including skeletal muscle $[11,13]$. To date LAP1B has not been reported to be involved in any disease.

Mutations in several genes that encode proteins of the nuclear envelope such as lamin A/C, lamin B, lamin B receptor, nesprin-1, nesprin-2 and emerin are known to cause defects of the nuclear envelope. This group of diseases known as nuclear envelopathies consist of a wide range of clinical syndromes, including muscular dystrophy, cardiomyopathy, lipodystrophy, mandibuloacral dysplasia, neuropathy, progeria, restrictive dermopathy, arthrogryposis, Pelger-Huet anomaly and leukodystrophy $[14,15]$.

Herein we report a consanguineous family with muscle weakness, atrophy and contractures in three affected individuals mapping the disease locus to chromosomal region 1q25. We identified a homozygous deletion in a novel nuclear-envelope related gene, torsinA-interacting protein 1 (TORIAIPI) encoding lamina-associated polypeptide 1B (LAP1B). The mutation located in the first exon is predicted to cause a premature stop codon and truncate the protein. Histological and molecular analyses demonstrate that the protein is absent in the patient's myonuclei and indicate a critical role for LAP1B in striated muscle tissue.

\section{Materials and methods}

\subsection{Patients}

The study included a multiplex consanguineous Turkish family with three affected individuals and healthy parents (Fig. 1A). The individuals affected by a phenotype with joint contractures were two siblings born to consanguineous parents and their paternal cousin, who was also born to consanguineous parents. Written informed consent to participate in the study was obtained from the patients and their family members. The study protocol was approved by the Hacettepe University Faculty of Medicine Ethics Committee (project number: TBK 11/11-02).

\subsection{Histopathological studies}

Muscle biopsy specimens obtained from the index case (IV:5) (Fig. 1A) at age 13 years and her brother (IV:2) (Fig. 1A) at age 36 years were evaluated. The shock-frozen muscle samples were stained with a battery of routine stains, including haematoxylin and eosin (H\&E), modified Gomori's trichrome (MGT), periodic acid-Schiff (PAS), Oil Red O (ORO), acid phosphatase, nicotine adenine dinucleotide tetrazolium reductase (NADH-TR), succinic dehydrogenase (SDH), cytochrome $\mathrm{C}$ oxidase (COX) and ATPase. For immunohistochemical examination by light microscopy, frozen sections $(8 \mu \mathrm{m})$ were incubated for $1 \mathrm{~h}$ at room temperature with antimerosin (1:20), anti-dystrophin (amino, rod and carboxyl domains, 1:2), anti-alpha sarcoglycan (1:20), anti-desmin (1:150), anti-emerin (1:20) and anti-lamin A/C (1:10) antibodies (all from Novocastra), and for $1 \mathrm{~h}$ with secondary antibodies. Dilutions and washing were performed using phosphate buffered saline.

For immunofluorescent staining; after fixation in 4\% paraformaldehyde for $10 \mathrm{~min}$ and washing in phosphate buffered saline (PBS), skeletal muscle sections $(7 \mu \mathrm{m})$ were blocked with 5\% bovine serum albumin in PBS for $1 \mathrm{~h}$ at room temperature. Incubation with the primary antibodies anti-LAP1B (1:400, Merck Millipore), anti-torsinA (1:50, Merck Millipore), anti-LULL1 (1:50, Sigma), anti-laminin alpha-2 (1:2000, Chemicon), anti-lamin B (1:50, Santa Cruz) and anti-PDI (1:100, Abcam) for $1 \mathrm{~h}$ at room temperature was followed by washing with PBS and labelling with Alexa Fluor 488 or 568 secondary antibody (1:1000, Molecular Probes) for $45 \mathrm{~min}$ at room temperature. After washing, DAPI staining, and mounting, the sections were examined under an inverted fluorescent 
A

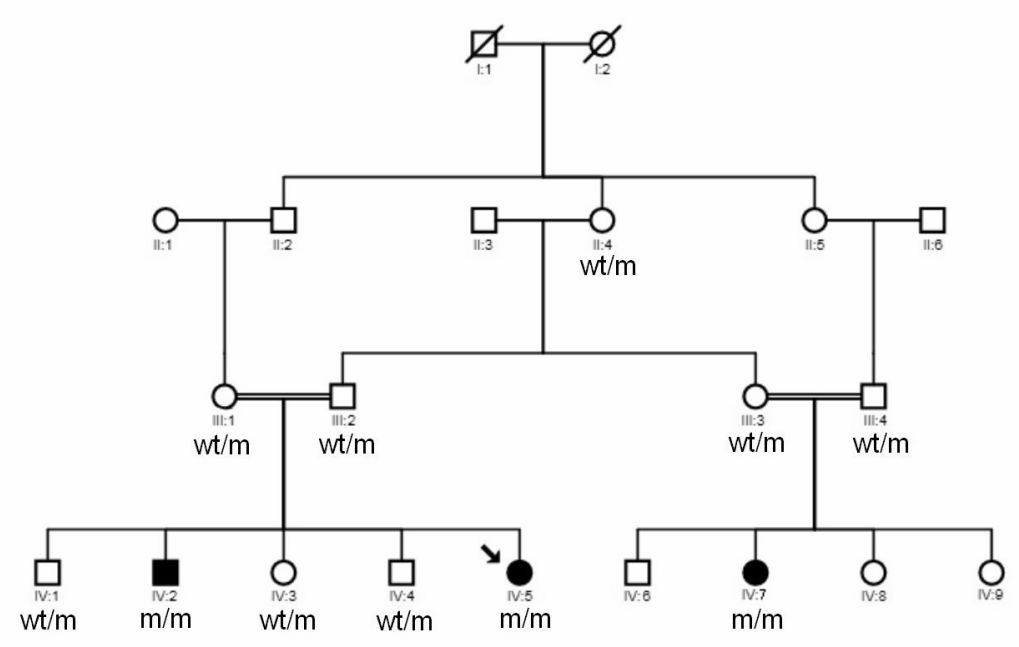

B

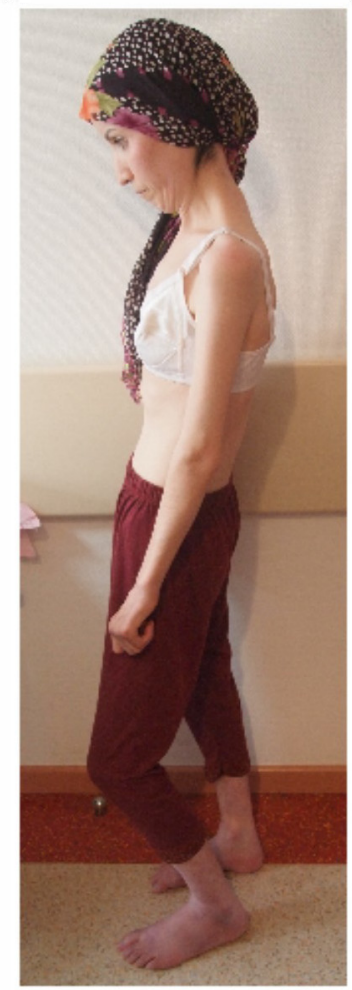

C

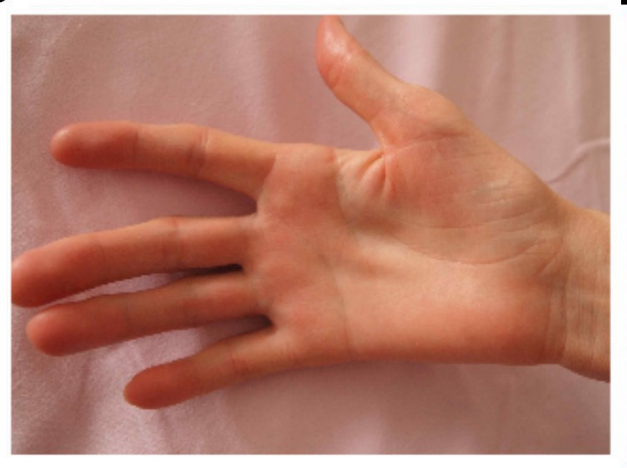

$\mathrm{E}$

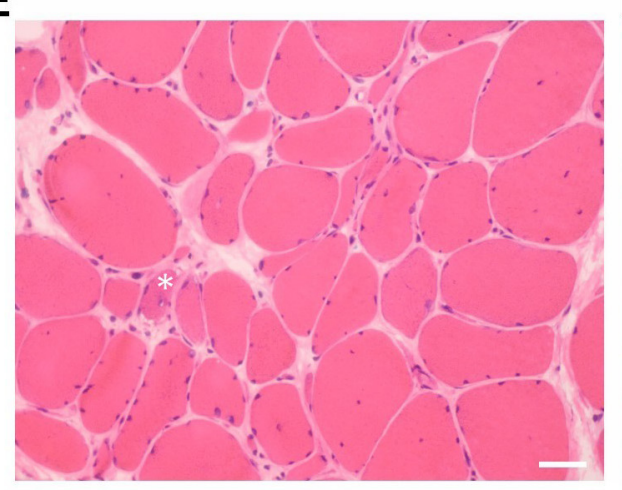

D

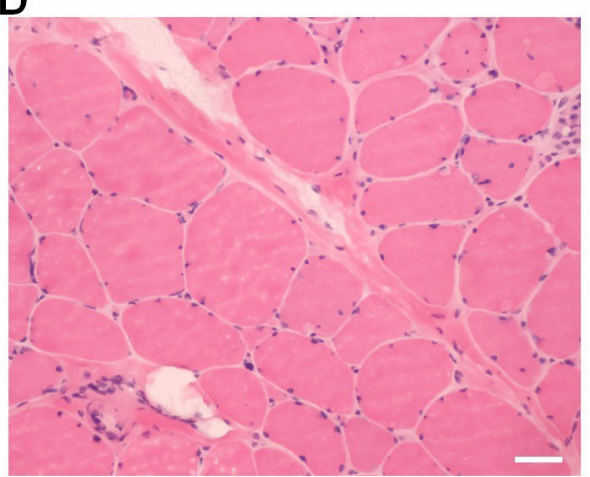

$\mathbf{F}$

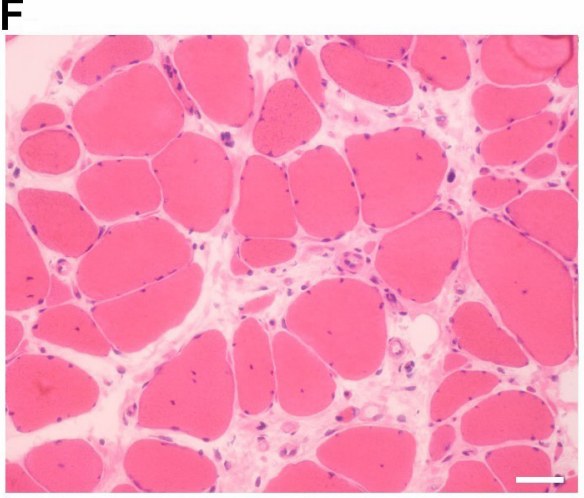

Fig. 1. Clinical and histopathological findings. (A) Pedigree of the family. DNA samples were obtained from individuals II:4, III:1, III:2, III:3, III:4, IV:1, IV:2, IV:3, IV:4, IV:5 and IV:7. Genome-wide homozygosity mapping was performed on affected members IV:2, IV:5, IV:7 and two healthy parents (III:1 and III:2) (wt: wild-type, m:mutant). (B and C) Clinical presentation of the index case (patient IV:5). (B) Thin appearance and (C) flexion contractures of the fingers. (D) Muscle biopsy specimen obtained from the index case (patient IV:5) shows variation in fibre size, increase in internalized nuclei and a mild increase in endomysial connective tissue. ( $\mathrm{E}$ and F) Muscle biopsy specimen obtained from patient IV:2 shows variation in fibre size, many atrophic fibres, increase in internal nuclei, rounded and a few angular fibres, rare subsarcolemmal vacuolar change $(*)$, and endomysial fibrosis - especially in the area of atrophic fibres. Scale bars represent $50 \mu \mathrm{m}$.

microscope (Leica DMIL) using appropriate excitation and barrier filters.

\subsection{Transmission electron microscopy}

Muscle samples were carefully dissected, fixed in $2.5 \%$ glutaraldehyde in phosphate buffer, rinsed in buffer and post-fixed in $1 \%$ osmium tetroxide in phosphate buffer at $4{ }^{\circ} \mathrm{C}$ for $2 \mathrm{~h}$. Specimens were dehydrated in graded series of ethanol to absolute ethanol in preparation for embedding in epon (EMS) using an automated tissue processor (Leica). Thin sections were stained with uranyl acetate and lead citrate, and ultrastructural muscular degeneration-regeneration criteria were analysed via a 
transmission electron microscope (Jeol) with an attached digital camera.

\subsection{Genetic and molecular analyses}

Genome-wide homozygosity mapping of the three affected members (IV:2, IV:5 and IV:7) and two healthy parents (III:1 and III:2) (Fig. 1A) was performed using the Affymetrix GeneChip Human Mapping 250K NspI Array. Genotype files (.chp) generated using Affymetrix GTYPE software were analysed via VIGENOS (Visual Genome Studio) software (Hemosoft, Ankara) [16]. Genome-wide haplotypes were constructed according to data obtained from the index case (IV:5). Genes located in the identified candidate region were identified using NCBI Map Viewer (Annotation Release 104) and the Ensembl database (Release 73.37).

DNA amplification and sequencing of all 10 exons and exon-intron boundaries of TORIAIPI (NM_015602.3) were performed using specific primers (see online Supplementary Table S1). The Big Dye Terminator cycle sequencing kit v.2.0 and the 3130 Genetic Analyser sequencing instrument (Applied Biosystems) were used for Sanger sequencing. The mutation was evaluated via public databases including dbSNP (http://www. ncbi.nlm.nih.gov/projects/SNP/), OMIM (http://www. ncbi.nlm.nih.gov/omim) and NHLBI Exome Variant Server (http://evs.gs.washington.edu/EVS/), and 154 healthy Turkish controls.

Total RNA was isolated from skeletal muscle biopsy specimens obtained from case IV:2 and three healthy controls using an RNeasy Fibrous Tissue kit (Qiagen), according to the manufacturer's instructions. RNA was converted into cDNA via reverse transcription using a QuantiTect Reverse Transcription kit (Qiagen), according to the manufacturer's instructions. Primers specific to TORIAIPI and to ACTB were used with SYBR Green JumpStart Taq ReadyMix (Sigma) and the experiments were performed in triplicate (see online Supplementary Table S2 for primers). Samples were analysed during the same run and the experiments were repeated 3 times for the same samples, and a no-template control was used during each run. $\mathrm{Ct}$ values were normalized to $A C T B$. Relative expression was obtained via the delta-delta $\mathrm{Ct}$ method using Corbett Rotor-Gene 6000 series software v.1.7 [17].

The total protein extracts concentration of patient IV:2 skeletal muscle, human control skeletal muscle, and liver and kidney samples were assessed using bicinchoninic acid assay. In total, $40 \mu \mathrm{g}$ of each protein extract was denatured via boiling and then subjected to $10 \%$ SDS/ PAGE. Following transfer to nitrocellulose membrane and blocking, immunoblotting was performed with primary antibodies anti-LAP1 (1:2500, a kind gift from Dr. William T. Dauer) or anti-LULL1 (1:750, Sigma) overnight at $4{ }^{\circ} \mathrm{C}$ and $\operatorname{IgG}(\mathrm{H}+\mathrm{L})$ HRP conjugate secondary antibody (1:3000, Molecular Probes) for $1 \mathrm{~h}$ at room temperature. Myosin bands shown by staining with Coomassie Brilliant Blue and immunoblot with HRP-conjugate anti-beta-actin (1:25,000, Abcam) were used for equal loading control. ChemiFast chemiluminescence substrate kit and GeneGnome chemiluminescence imaging instrument (Syngene) were used for chemiluminescence detection.

\section{Results}

\subsection{Clinical characteristics}

The index case (IV:5) (Fig. 1B) was a 29-year-old female that had onset of proximal muscle weakness at age 7 years. Currently, she can walk unaided but cannot rise from floor. She has a thin appearance with flexion contractures of the proximal and distal interphalangeal hand joints along with a mild rigid spine in the cervical region (Fig. 1B and $\mathrm{C}$ ). Her serum creatine kinase (CK) level was $89 \mathrm{U} \mathrm{L}^{-1}$ (normal range: $26-126 \mathrm{U} \mathrm{L}^{-1}$ ). Electrocardiogram (ECG) and echocardiogram performed at age 29 years were normal. She had restriction in her pulmonary function: FEV1 (forced expiratory volume in $1 \mathrm{~s}$ ) was $43 \%$.

Her 36 year-old brother (IV:2) was similarly affected. He had his first symptoms at the age of 17 years as difficulty in climbing stairs, which progressed to tiring in legs and arms, and proximal and marked distal muscle weakness within years. In addition to muscle weakness, he developed contractures with spine, ankle and hand joint involvement. There was marked atrophy in his interossei, abductor pollicis brevis, and medial gastrocnemius muscles. He could walk unaided for a distance of only $40 \mathrm{~m}$. His serum CK level was $483 \mathrm{UL}^{-1}$. Electromyography showed a disseminated myogenic pattern, even in the most atrophied dorsal interossei muscles. His cardiac evaluation performed at age 36 showed mild diastolic and systolic dysfunction with an ejection fraction of $57 \%$ via echocardiography. Electrocardiogram was normal and $24 \mathrm{~h}$ Holter monitoring revealed normal sinus rhythm with ventricular extrasystoles. Angiotensin-converting enzyme inhibitor and beta blocker were initiated. He had moderate restrictive respiratory function, with FEV1 of $63 \%$ via spirometry. The siblings have a paternal cousin (IV:7) that is also affected; however, she has not been clinically investigated.

\subsection{Histopathological findings}

The muscle biopsy specimen from the vastus lateralis muscle of the index case (IV:5) at age 13 years (Fig. 1D) showed mild dystrophic features with variation in fibre size, increase in internal nuclei and endomysial connective tissue, and degeneration and phagocytosis in a few fibres. Merosin, dystrophin, and alpha sarcoglycan immunohistochemistry were normal. Vastus lateralis muscle biopsy of the affected brother (IV:2) at age 35 years (Fig. 1E and F) showed more prominent variation in fibre size and 
increase in fibres with internal nuclei. In some areas very small fibres and a prominent increase in endomysial connective tissue were observed (Fig. 1F). Some of the atrophic fibres had relatively large nuclei that resembled nuclear clumps of denervated fibres. A few fibres exhibited vacuolar degeneration and some fibres exhibited basophilic degeneration. There were no rimmed vacuoles. Type 2 fibres predominated and atrophic fibres were of both fibre types (type 1 and type 2). There was no regeneration, inflammation, increase in lipid or glycogen, or lysosomal activity. Lamin $\mathrm{A} / \mathrm{C}$ and emerin immunohistochemistry were normal, whereas some fibres exhibited irregular desmin deposition (see online Supplementary Fig. S1A). Further analyses were performed using the muscle biopsy specimen of this patient (IV:2).

Immunofluorescent staining showed a substantial loss of LAP1B in the patient IV:2's muscle, with a few scattered nuclei staining positive (Fig. 2A). Double-labelling with anti-LAP1B and anti-laminin alpha-2 antibodies showed that the positive nuclear staining for LAP1B was not within the muscle fibres but in the endomysial area (Fig. 2B). Immunostaining of the patient's muscle with anti-lamin B, anti-LULL1 and anti-torsinA antibodies was similar to control muscle (see online Supplementary Fig. S1B-D). TorsinA and LULL1 appeared to be distributed in the sarcoplasmic reticulum in both patient and control muscle.

A
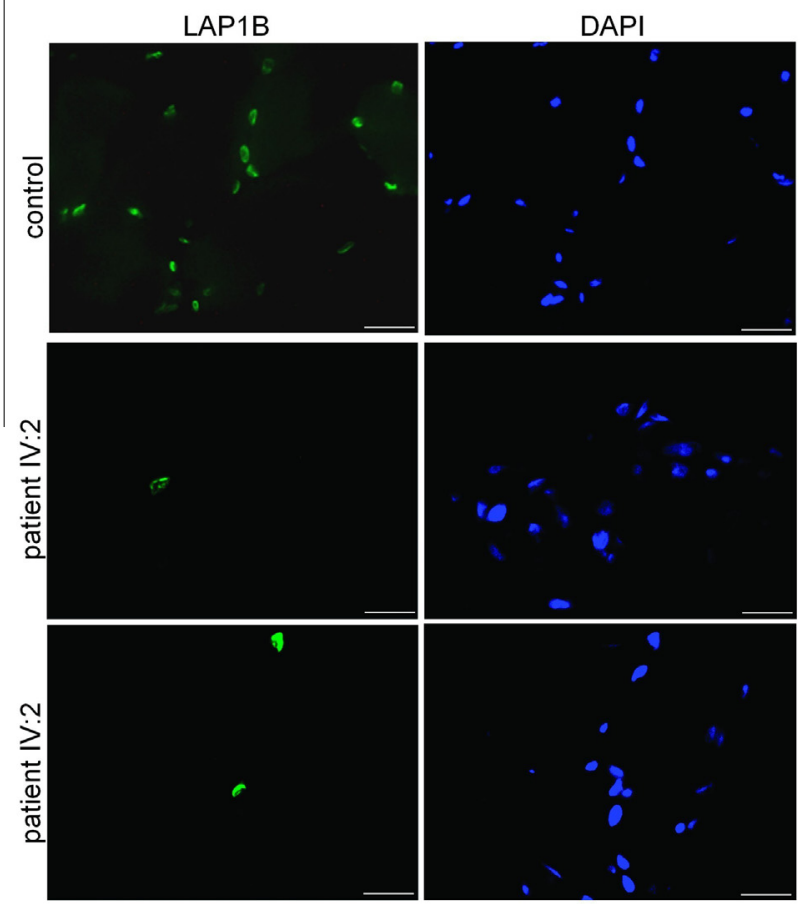

\subsection{Ultrastructural findings}

Transmission electron microscopy exhibited intact cytoplasmic sarcomeric organization with aligned Z bands, good myofibril integrity and no sarcolemmal disturbance at the ultrastructural level. A few centrally located nuclei were noted and some nuclei exhibited varying degrees of chromatin condensation and aggregation. Nuclear fragmentation, chromatin bleb formation and naked chromatin devoid of membrane were evident as a consequence of nuclear membrane disruption and karyoplasmic leakage into the sarcoplasmic compartment (Fig. 3A-E). Variable degrees of nuclear deformation were noted in about $40-60 \%$ of the cell nuclei being trimmed for TEM. Vacuolar fibres were not detected at the ultrastructural examination.

\subsection{Genetic and molecular studies}

Because of the initial limb-girdle muscular dystrophy-like diagnosis, all known LGMD2 loci were previously excluded via linkage analysis. Genome-wide homozygosity mapping showed a single homozygous haplotype shared by the three affected individuals on chromosomal region 1q25 (Fig. 4A). This region spanning approximately $1.8 \mathrm{Mb}$ resided between SNP markers rs316265 (SNP_A-1973844) and rs16855014 (SNP_A-1802874). According to NCBI Map Viewer (Annotation Release 104) and Ensembl

B

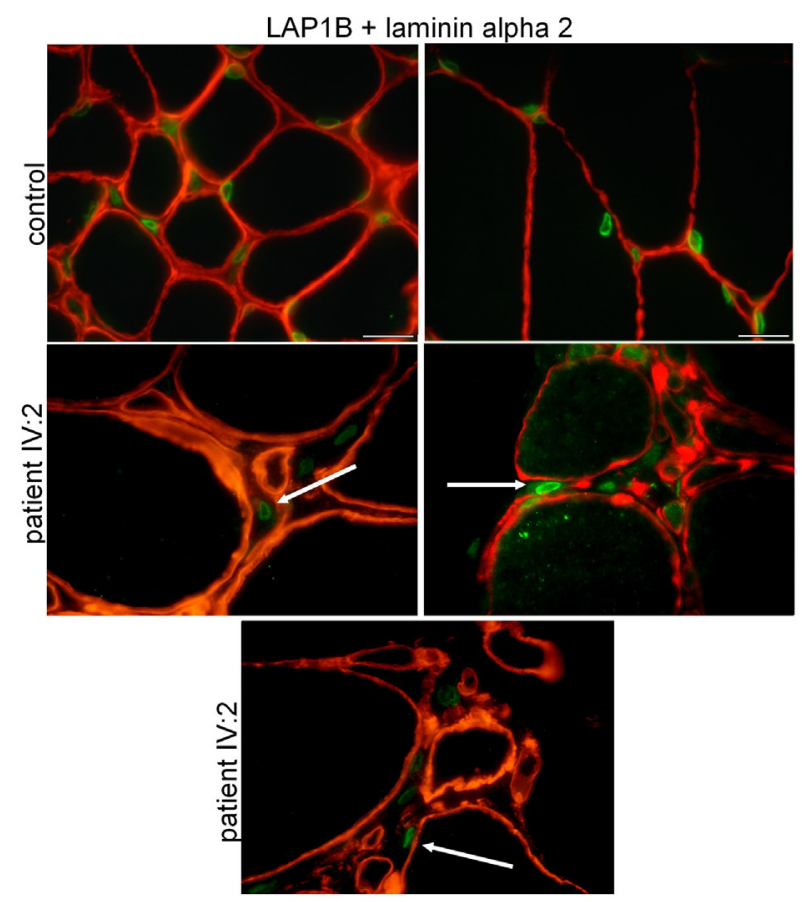

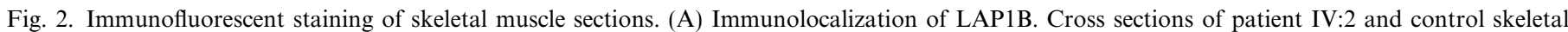

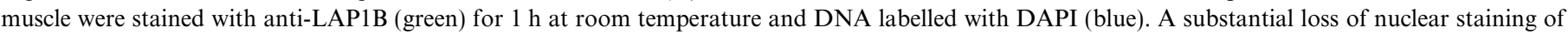

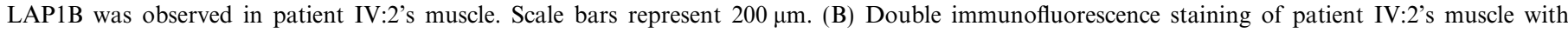

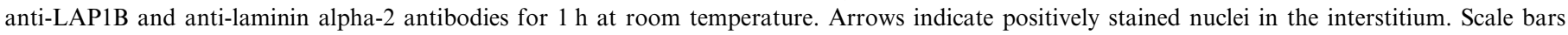
represent $200 \mu \mathrm{m}$. 

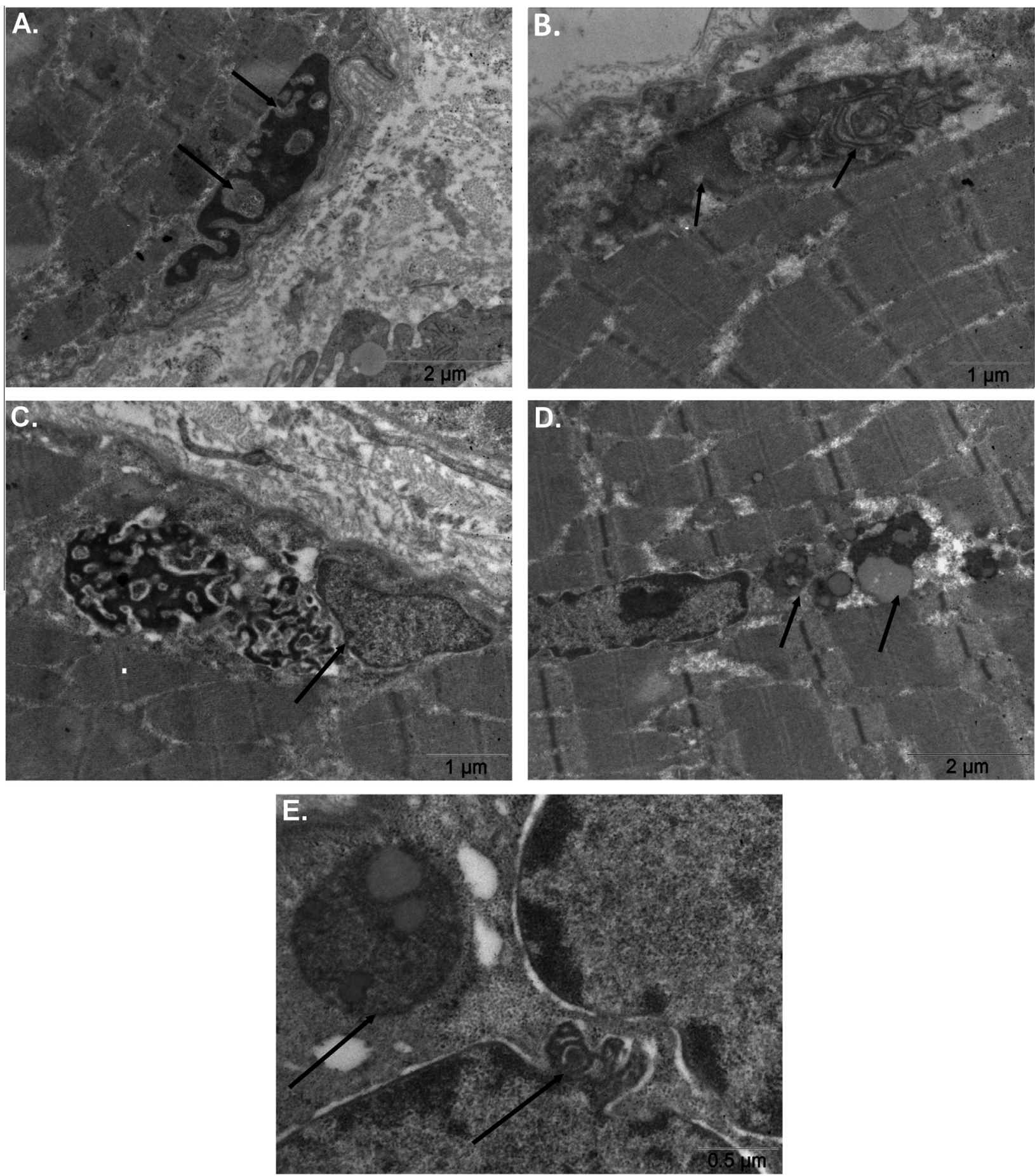

Fig. 3. Ultrastructural alterations of the nuclear envelope. (A) Nuclear fragmentation and chromatin bleb formation (arrow). (B) Nuclear membrane disintegration and intranuclear canalicule formation (arrows). (C) A highly fragmented, degenerated nucleus, and adjacent naked chromatin (arrow). (D) Nuclear disintegration with degenerating naked chromatin fragments (arrows). (E) High magnification of the crumbling and disintegration of the nuclear envelope and subsequent naked chromatin fragment formation (arrows).

Genome Browser (Release 73.37) databases, this region between $178.100 .703 \mathrm{bp}$ and $179.925 .538 \mathrm{bp}$ harboured fifteen protein-coding genes among which TORIAIPI (MIM 614512) was selected as a candidate gene (see online Supplementary Table S3). This gene encodes a type-2 integral membrane protein located in the inner nuclear membrane, LAP1B. Because other proteins of the nuclear membrane such as lamin $\mathrm{A} / \mathrm{C}$, emerin, nesprin-1 and nesprin-2 are known to cause muscular dystrophy phenotypes, TORIAIP1 was selected as a possible novel nuclear envelopathy-related gene $[14,15]$. DNA sequencing of TORIAIP1 from the genomic DNA of affected individuals IV:2, IV:5 and IV:7 showed that there was a homozygous $\mathrm{G}$ deletion at position c.186 (c.186delG) in 

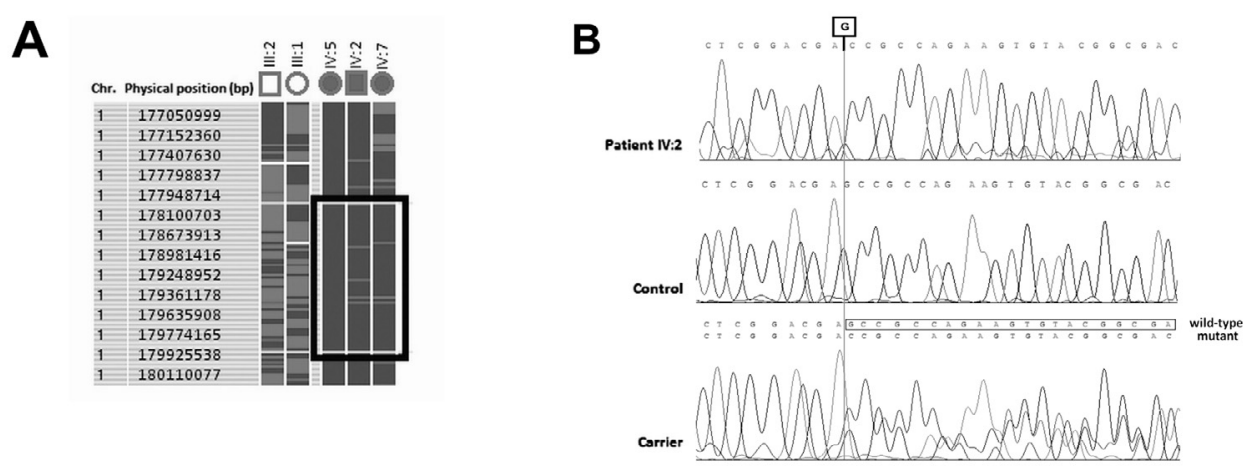

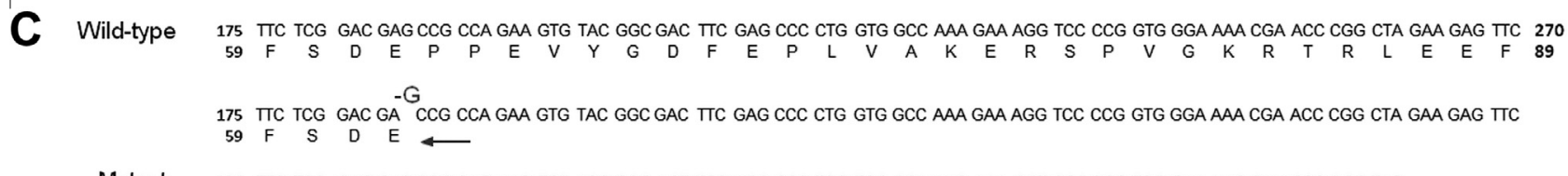

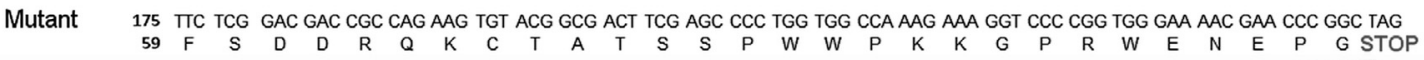

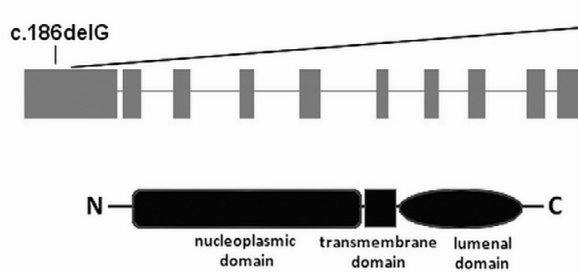

Fig. 4. Homozygosity mapping and mutation analysis. (A) Representation of homozygosity mapping data. Genome-wide haplotypes were constructed according to data obtained from the index case IV:5. Homozygous genotypes identical to the genotypes of the index case (IV:2) are shown in dark grey, heterozygous genotypes appear in light grey. SNPs that are non-informative due to heterozygous genotypes in parent-child trios appear in white. The single homozygous segment shared by the three affected individuals spanned approximately $1.8 \mathrm{Mb}$ on chromosome 1 and is marked in the rectangle. (B) DNA sequence chromatograms show homozygous c.186delG mutation in patient IV:2, heterozygous mutation in his mother (III:1) and wild-type sequence in a control subject. (C) The mutation is predicted to cause a frameshift and a premature stop codon in the first exon.

the first exon of the gene, which was predicted to cause a frameshift leading to a premature stop codon (Fig. 4B and C). As a result, the 583 amino-acid protein is predicted to be truncated to a non-functional protein 83 amino-acids in length (p.E62fsTer25). The healthy parents (II:4, III:1, III:2, III:3 and III:4) and siblings (IV:1, IV:3 and IV:4) were heterozygous carriers and allele segregation in the family was consistent with autosomal recessive inheritance. The mutation was not detected in 154 healthy controls of Turkish origin or in public SNP databases.

Expression of TOR1AIP1 mRNA was assessed via qRT-PCR. Relative quantitation of TORIAIPI showed that the level of expression in the skeletal muscle of patient IV:2 was 5-fold lower than in three control samples (Fig. 5A). The analysis of the expression profile of the LAP1B protein via Western blotting showed a total of two specific bands (Fig. 5B). The $66.3 \mathrm{kDa}$ band corresponding to LAP1B detected in control skeletal muscle, liver, and kidney samples was absent in the muscle of patient IV:2. An additional band with a molecular weight of approximately $50 \mathrm{kDa}$ present in all samples appeared to be more abundant in the muscle of patient IV:2 than in control muscle (Fig. 5B, *). Immunoblotting with anti-LULL1 antibody showed that expression of LULL1 in the patient IV:2's muscle was greater than that in control muscle (Fig. 5B).

\section{Discussion}

We identified a novel nuclear envelope disease caused by a homozygous mutation in TORIAIP1, encoding LAP1B, presenting as a myopathy with contractures. The study patients developed proximal and severe distal weakness and atrophy, rigid spine and contractures in the ankle and small hand joints, as well as cardiac and respiratory involvement. The extent of weakness and atrophy in the distal muscles in patient IV:2 was similar to that associated with longstanding neuropathy; however, electromyography in these muscles showed myopathic potentials. Although there were some fibres resembling nuclear clumps in his muscle biopsy from the vastus lateralis, the histopathological features were mainly compatible with a myopathic process. In cases with muscle weakness, spinal rigidity, contractures and cardiac involvement, lamin A/C and emerin related EDMD must be considered in the differential diagnosis. However, although the patients we report here had rigidity of the spine and ankle contractures, they did not have elbow contractures and the 
A
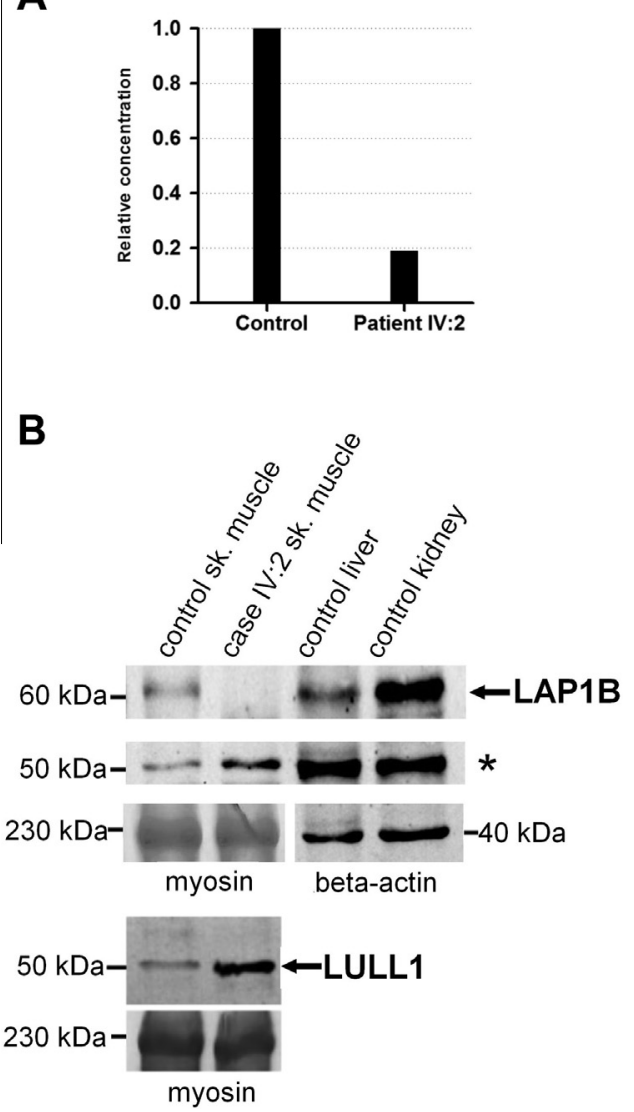

Fig. 5. Expression analysis. (A) Analysis of TOR1AIP1 mRNA expression via qRT-PCR. Primers bridging exon 1 and exon 2 of TORIAIP1 and primers specific to ACTB were used and the experiments were performed in triplicate. $\mathrm{Ct}$ values for the control samples and the patient muscle sample were normalized to $A C T B$. Relative expression was obtained using the delta-delta $\mathrm{Ct}$ method [17]. Expression of TORIAIP1 mRNA was 5-fold lower in patient IV:2 muscle than in control samples. (B) Expression analysis of LAP1B protein based on Western blotting. Protein extracts $(40 \mu \mathrm{g})$ of patient IV:2 and control skeletal muscle, and control liver and kidney samples were immunoblotted via incubation of primary anti-LAP1 antibody overnight at $4{ }^{\circ} \mathrm{C}$ and HRP-conjugated secondary antibody for $1 \mathrm{~h}$ at room temperature. Myosin bands shown via staining with Coomassie Brilliant Blue and immunoblot with anti-beta-actin antibody were used for equal loading control. A ChemiFast chemiluminescence substrate kit (Syngene) and GeneGnome chemiluminescence imaging instrument (Syngene) were used for chemiluminescence detection using 5-s exposure. The $66.3 \mathrm{kDa}$ band corresponds to LAP1B. One additional band of approximately $50 \mathrm{kDa}$ was observed $(*)$. Analysis of LULL1 expression via western blotting showed that expression of LULL1 in the patient muscle was higher than in the control muscle.

degree of atrophy, especially severe atrophy in the hands and finger contractures were not compatible with any known EDMD phenotype $[4,18]$. In addition, pedigree was not compatible with an X-linked disorder. Among other diseases causing a contractural phenotype, marked distal atrophy and cardiac involvement were unusual for collagen VI related myopathies, joint laxity was not present from the history or at physical examination [19]. Presence of cardiac features, distal involvement with atrophy and contractures, and absence of early respiratory involvement were not consistent with selenoprotein related disease [20]. Ultrastructural alterations of the nuclear envelope observed in the patient IV:2's muscle, such as disruption of the nuclear membrane, and chromatin condensation and aggregation are consistent with the nuclear envelope morphological abnormalities observed in laminopathies (Fig. 3A-E) [21,22]. This pattern is distinguishable from myopathic features which exhibit degenerative ultrastructural changes starting from the myocytic plasma membrane [23].

LAP1B encoded by TORIAIP1 is type-2 integral membrane protein located in the inner nuclear membrane binding both A- and B-type lamins and involved in the regulation of torsinA ATPase, a member of AAA + ATPase family (ATPases associated with a variety of cellular activities) known to be responsible for the severe movement disorder DYT1 dystonia [7-12]. TorsinA is diffusely distributed throughout the endoplasmic reticulum and the nuclear envelope continuity but some cell types exhibit a preference for the nuclear envelope [24,25]. Luminal domain-like LAP1 (LULL1) encoded by TOR1AIP2 is located in the endoplasmic reticulum and shares homologous C-terminal regions with LAP1 allowing interaction with ER-located torsinA [11,24]. LAP1 and LULL1 appear to have arisen from a gene duplication [11].

The c.186delG mutation in TOR1AIP1 is predicted to cause a premature stop codon during translation, truncating the 584 amino acid LAP1B protein to a protein that is 86 amino acids in length (Fig. 4C). It is predicted that the resulting mutant RNA is eliminated by nonsense-mediated decay and that the truncated protein is non-functional. Because no other isoform is described in humans, we expected an absence of LAP1B in all tissues, but our observations suggest that one LAP1 isoform - other than that expressed in myonuclei-might be present in endomysial cells (Fig. 2B). Because the endomysial cell population is greater in the dystrophic muscle of the patient IV:2 than in the control muscle, this isoform might correspond to the additional band of around $50 \mathrm{kDa}$ that was more abundant in the patient IV:2's muscle than in control muscle, but was also present in liver and kidney samples (Fig. 5B,*).

Constitutive knockout of LAP1 in mice causes perinatal lethality; however, a very recent study reported that the conditional deletion of LAP1 from mouse striated muscle leads to muscular dystrophy whereas LAP1 deletion from hepatocytes does not affect liver function [13,26]. This model supports the notion that LAP1B has a specific role in striated muscle and represents a functional validation of the effect of LAP1B deficiency in human striated muscle. Data on the expression of LAP1 in different species show that three different isoforms of LAP1 are present in most tissues [7,9]. The present results show that the $66.3 \mathrm{kDa}$ isoform in human has a specific and critical role in muscle tissue although it is also expressed in the liver and kidneys. 
The recent finding that LAP1 interacts with emerin, which causes the EDMD phenotype, strongly suggests that LAP1 is as essential as emerin in striated muscle function; however, the lack of a pathological phenotype in emerin-null mice indicates that other factors are involved in the function of the same proteins in different species [26]. It is proposed that a higher level of expression of LAP1 may compensate for the lack of emerin in emerin deficient mice [26]. The same study also reported that dislocalisation of emerin and lamin $\mathrm{A} / \mathrm{C}$ in nuclear foci was noted in approximately $10 \%$ of myofibres isolated from the skeletal muscle of LAP1 muscle-specific conditional knockout mice [26]. In the present study, immunostaining of lamin A/C, lamin B and emerin did not show any difference of staining pattern between patient IV:2 and control samples (see online Supplementary Fig. S1A and B).

In contrast to LAP1 knockout mice exhibiting perinatal lethality, loss of function of LAP1B in humans appears to affect only muscle tissue, and a possible explanation is that variation in the level of expression of the different LAP1 isoforms observed in mouse and human tissue, might lead to different phenotypes in the case of loss of function of LAP1 [26]. In the presented case, only the $66.3 \mathrm{kDa}$ isoform was absent in muscle fibres. Another hypothesis is that similar to emerin and LAP1 interaction, LULL1 might have a compensatory effect on the phenotype in the absence of LAP1. Although no data on LULL1 expression are provided in the mouse model, overexpression of LULL1 in human might be related to the relatively milder phenotype observed in humans, as compared to mice.

Although knockdown of LAP1 or LULL1 does not seem to affect expression or localization of torsinA, mice lacking LAP1 exhibit perinatal lethality and LAP1 deficiency causes nuclear membrane abnormalities similar to those observed in torsinA-null cells $[13,27]$. On the other hand, overexpression of LAP1 and LULL1 recruits torsinA to the nuclear envelope $[11,28]$. A recent study reported that ATPase activity of torsinA is induced in the presence of LAP1 or LULL1 and that LAP1 and LULL1 are regulatory cofactors of torsinA [12]. In agreement with the literature, in the present study we did not observe any change in the localization of torsinA or LULL1 in the absence of LAP1B, as compared to control muscle (see online Supplementary Fig. S1B and C); however, an increase in expression of LULL1 was observed in the patient IV:2 (Fig. 5B). It was suggested that LAP1 and LULL1 have similar modes of action on torsinA and that regulation of its ATPase activity is dependent on the dose of its cofactors [12]. Furthermore, distribution of torsinA might be regulated by the relative abundance of its binding partners in the ER and the nuclear envelope [24]. According to these hypotheses, overexpression of LULL1 observed in the patient IV:2 might have been associated with the absence of LAP1B and these two proteins might have a compensatory effect on each other.
Similar to other known nuclear envelopathies, in the presented cases of TORIAIPI mutation there is a tissue-specific phenotype caused by a mutation in a gene that is apparently expressed ubiquitously [29]. Although the exact mechanism of this tissue specificity remains unknown, one model proposes that the nuclei of some cell types, such as skeletal and cardiac muscle in the presented cases are prone to an important mechanical stress and are more susceptible than other cells [30]. The absence of LAP1B in muscle might influence the structural and mechanical stability of the nuclear envelope due to impaired binding to the nuclear lamina. LAP1 is not the sole torsin A partner localized in the nuclear envelope. Other torsinA-binding partners, including components of the LINC complex SUN1, SUN2 and nesprins, have been described $[27,31,32]$. A possible consequence of LAP1B deficiency might be altered interaction of cytoskeletal components with the nuclear envelope via misbinding of torsinA to other partners in the absence of LAP1B. Although we did not observe any mislocalisation of torsinA, its function might be altered without any noticeable impact on its localization.

Another hypothesis is that defects of proteins in the nuclear envelope might alter tissue-specific gene expression patterns $[29,30]$. It is known that lamin A/C and emerin are involved in muscle transcriptional pathways [33-37]. One study proposed that LAP1 might bind indirectly to mitotic chromosomes via a linker protein [9]. If LAP1B has such a chromatin or transcriptional regulator binding function, the disturbance of heterochromatin organization observed in the presented patient might influence expression of genes specific to striated muscle.

In conclusion, we described a new autosomal recessive nuclear envelope disease caused by a homozygous mutation in exon 1 of TORIAIP1 encoding LAP1B. The clinical presentation of the present study patients included proximal limb-girdle weakness during childhood that progressed to distal weakness and contractures, spinal rigidity and cardiomyopathy in adulthood. LAP1B is an integral membrane protein of the inner nuclear membrane known to bind A- and B-type lamins and regulate torsinA ATPase activity. The muscle-restricted phenotype suggests that LAP1B has a specific role in the nuclear envelope of cardiac and skeletal muscle tissues. In addition, overexpression of LULL1 in the absence of LAP1 might support the hypothesis that these two proteins act via the same pathway. The present study expands the spectrum of genes associated with nuclear envelopathies and highlights the critical function of LAP1B in human striated muscle tissue.

\section{Funding}

This study was supported by the Scientific and Technological Research Council of Turkey [Project Number: 112 S271 to P.D.]. 


\section{Acknowledgements}

We are very grateful to the patients and their family for agreeing to participate in this study. We thank Dr. Gisèle Bonne for critical review of the manuscript.

\section{Appendix A. Supplementary data}

Supplementary data associated with this article can be found, in the online version, at http://dx.doi.org/10.1016/ j.nmd.2014.04.007.

\section{References}

[1] Emery AE. The muscular dystrophies. BMJ 1998;317:991-5.

[2] Guglieri M, Straub V, Bushby K, Lochmüller H. Limb-girdle muscular dystrophies. Curr Opin Neurol 2008;21:576-84.

[3] Muntoni F, Lichtarowicz-Krynska EJ, Sewry CA, et al. Early presentation of X-linked Emery-Dreifuss muscular dystrophy resembling limb-girdle muscular dystrophy. Neuromuscul Disord 1998;8:72-6.

[4] Bonne G, Mercuri E, Muchir A, et al. Clinical and molecular genetic spectrum of autosomal dominant Emery-Dreifuss muscular dystrophy due to mutations of the lamin A/C gene. Ann Neurol 2000;48:170-80.

[5] Udd B. Distal myopathies - new genetic entities expand diagnostic challenge. Neuromuscul Disord 2012;22:5-12.

[6] Selcen D. Myofibrillar myopathies. Neuromuscul Disord 2011;21:161-71.

[7] Senior A, Gerace L. Integral membrane proteins specific to the inner nuclear membrane and associated with the nuclear lamina. J Cell Biol 1988;107:2029-36.

[8] Kondo Y, Kondoh J, Hayashi D, et al. Molecular cloning of one isotype of human lamina-associated polypeptide 1s and a topological analysis using its deletion mutants. Biochem Biophys Res Commun 2002;294:770-8.

[9] Foisnier R, Gerace L. Integral membrane proteins of the nuclear envelope interact with lamins and chromosomes, and binding is modulated by mitotic phosphorylation. Cell 1993;73:1267-79.

[10] Maison C, Pyrpasopoulou A, Theodoropoulos PA, Georgatos SD. The inner nuclear membrane protein LAP1 forms a native complex with B-type lamins and partitions with spindle-associated mitotic vesicles. EMBO J 1997;16:4839-50.

[11] Goodchild RE, Dauer WT. The AAA + protein torsinA interacts with a conserved domain present in LAP1 and a novel ER protein. J Cell Biol 2005; 168:855-62.

[12] Zhao C, Brown RS, Chase AR, Eisele MR, Schlieker C. Regulation of torsin ATPases by LAP1 and LULL1. Proc Natl Acad Sci USA 2013;110:E1545-54

[13] Kim CE, Perez A, Perkins G, Ellisman MH, Dauer WT. A molecular mechanism underlying the neural-specific defect in torsinA mutant mice. Proc Natl Acad Sci USA 2010;107:9861-6.

[14] Worman HJ, Östlund C, Wang Y. Diseases of the nuclear envelope. Cold Spring Harb Perspect Biol 2010;2:a000760.

[15] Zhang Q, Bethmann C, Worth NF, et al. Nesprin-1 and -2 are involved in the pathogenesis of Emery-Dreifuss muscular dystrophy and are critical for nuclear envelope integrity. Hum Mol Genet 2007;16:2816-33.

[16] Gundesli H, Talim B, Korkusuz P, et al. Mutation in exon 1f of PLEC, leading to disruption of plectin isoform 1f, causes autosomal-recessive limb-girdle muscular dystrophy. Am J Hum Genet 2010;87:834-41.
[17] Livak KJ, Schmittgen TD. Analysis of relative gene expression data using real-time quantitative PCR and the $2(-\mathrm{D} D \mathrm{D}(\mathrm{T}))$ method. Methods 2001;25:402-8.

[18] Puckelwartz M, McNally EM. Emery-Dreifuss muscular dystrophy. Handb Clin Neurol 2011;101:155-66.

[19] Bönnemann CG. The collagen VI-related myopathies Ullrich congenital muscular dystrophy and Bethlem myopathy. Handb Clin Neurol 2011;101:81-96.

[20] Schara U, Kress W, Bönnemann CG, et al. The phenotype and long-term follow-up in 11 patients with juvenile selenoprotein N1-related myopathy. Eur J Paediatr Neurol 2008;12(3):224-30.

[21] Sewry CA, Brown SC, Mercuri E, et al. Skeletal muscle pathology in autosomal dominant Emery-Dreifuss muscular dystrophy with lamin A/C mutations. Neuropathol Appl Neurobiol 2001;27:281-90.

[22] Arbustini E, Pilotto A, Repetto A, et al. Autosomal dominant dilated cardiomyopathy with atrioventricular block: a lamin A/C defect-related disease. J Am Coll Cardiol 2002;39:981-90.

[23] Cetin N, Balci-Hayta B, Gundesli H, et al. A novel desmin mutation leading to autosomal recessive limb-girdle muscular dystrophy: distinct histopathological outcomes compared with desminopathies. J Med Genet 2013:1-7.

[24] Naismith TV, Dalal S, Hanson PI. Interaction of TorsinA with its major binding partners is impaired by the dystonia-associated deltaGAG deletion. J Biol Chem 2009;284:27866-74.

[25] Giles LM, Chen J, Li L, Chin L-S. Dystonia-associated mutations cause premature degradation of torsinA protein and cell-type-specific mislocalization to the nuclear envelope. Hum Mol Genet 2008;17: 2712-22.

[26] Shin JY, Méndez-López I, Wang Y, et al. Lamina-associated polypeptide-1 interacts with the muscular dystrophy protein emerin and is essential for skeletal muscle maintenance. Dev Cell 2013;26:1-13.

[27] Jungwirth M, Kumar D, Jeong D, Goodchild R. The nuclear envelope localization of DYT1 dystonia torsinA-DeltaE requires the SUN1 LINC complex component. BMC Cell Biol 2011;12:24.

[28] Vander Heyden AB, Naismith TV, Snapp EL, Hodzic D, Hanson PI. LULL1 retargets TorsinA to the nuclear envelope revealing an activity that is impaired by the DYT1 dystonia mutation. Mol Biol Cell 2009;20:2661-72.

[29] Worman HJ, Courvalin J-C. How do mutations in lamins A and C cause disease? J Clin Invest 2004;113:349-51.

[30] Roux KJ, Burke B. Nuclear envelope defects in muscular dystrophy. Biochim Biophys Acta 2007;1772:118-27.

[31] Nery CF, Zeng J, Niland BP, et al. TorsinA binds the KASH domain of nesprins and participates in linkage between nuclear envelope and cytoskeleton. J Cell Sci 2008:121:3476-86.

[32] Gerace L. TorsinA and torsion dystonia: unraveling the architecture of the nuclear envelope. Proc Natl Acad Sci USA 2004;101:8839-40.

[33] Holaska JM, Lee KK, Kowalski AK, Wilson KL. Transcriptional repressor germ cell-less (GCL) and barrier to autointegration factor (BAF) compete for binding to emerin in vitro. J Biol Chem 2003;278:6969-75.

[34] Haraguchi T, Holaska JM, Yamane M, et al. Emerin binding to Btf, a death-promoting transcriptional repressor, is disrupted by a missense mutation that causes Emery-Dreifuss muscular dystrophy. Eur J Biochem 2004;271:1035-45.

[35] Melcon G, Kozlov S, Cutler DA, et al. Loss of emerin at the nuclear envelope disrupts the Rb1/E2F and MyoD pathways during muscle regeneration. Hum Mol Genet 2006;15:637-51.

[36] Markiewicz E, Ledran M, Hutchison CJ. Remodelling of the nuclear lamina and nucleoskeleton is required for skeletal muscle differentiation in vitro. J Cell Sci 2005;118:409-20.

[37] Favreau C, Higuet D, Courvalin JC, Buendia B. Expression of a mutant lamin A that causes Emery-Dreifuss muscular dystrophy inhibits in vitro differentiation of $\mathrm{C} 2 \mathrm{C} 12$ myoblasts. Mol Cell Biol 2004:24:1481-92. 